\title{
Finding Our Way
}

\author{
Amy Bauer, RN, BSN
}

\begin{abstract}
Long before I got pregnant, my husband and I began to prepare for the kind of birth I had always imagined for myself: peaceful, normal, and without medication or unnecessary medical intervention. In the fall of 2008, I got pregnant. At that same time, I started a new career as a labor and delivery nurse in a hospital that specializes in high-risk labor and birth. My daughter's journey into this world turned out to be the beautiful normal experience we had always imagined. This special experience also helped shape the kind of care I offer as a new labor and delivery nurse.
\end{abstract}

The Journal of Perinatal Education, 20(1), 6-7, doi: 10.1891/1058-1243.20.1.6

Keywords: normal birth, natural birth, labor and delivery nursing

Do you have a birth story that illustrates the power of childbirth and encourages women to give birth with confidence? We invite you to submit your story for possible publication in The Journal of Perinatal Education. Please send your story or query to Judith Lothian, Associate Editor(Jalothian@aol.com).

When I got pregnant during the fall of 2008, my husband and I set right to work to prepare for our child's birth. Long before getting pregnant, I knew what kind of birth experience I wanted to have. We researched birth centers in our area, identified childbirth education and lactation classes, and talked at length about what it means to have a natural, normal birth.

Throughout my pregnancy, I worked the night shift as a new labor and delivery nurse at a hospital that specializes in high-risk pregnancy and birth. At this hospital, a natural, normal birth is a rare event. All too often, physicians meddle with the process in an effort to "actively manage" women's labor. Artificial rupture of membranes and Pitocin are routinely

I confided in fellow nurses who were strong advocates of natural, normal birth and poured over written birth stories that illustrated the beauty and power of natural birth. used to hurry labor along, at times without strong medical indication. On top of that, nearly $95 \%$ of the hospital's patients opt for epidural anesthesia, and approximately $41 \%$ of births are by cesarean surgery. New to nursing and now preparing for my own labor, I felt uncertain how, as a new labor and delivery nurse, I could best support women in labor and birth in this environment. I also began to wonder if my body would know what to do in labor. As my due date neared, I shared these concerns with Katie, my midwife, who gave me a great piece of advice: While working in the labor and delivery unit, I was to (a) visualize my own labor and birth every day, (b) surround myself with positive stories about natural labor and birth, and (c) keep my "nursing brain" out of my own labor and birth when labor began. I did just that-I confided in fellow nurses who were strong advocates of natural, normal birth and poured over written birth stories that illustrated the beauty and power of natural birth. At the risk of feeling a bit silly, I also recited positive affirmations each day to 
remind myself that my baby would find her way and that I could, in fact, trust my body when labor began.

In the early morning hours of June 27, 2009, my labor began with mild, irregular contractions. It was a beautiful, yet uneventful labor, lasting nearly 19 hours from start to finish. In the beginning, distraction helped. In between contractions, I sneaked out of bed at 4:00 a.m. to write thank-you notes for a recent baby shower. Rhythm also proved to be helpful. Later that morning, when labor became more intense, I relied heavily on my husband to literally rock me back and forth through each contraction. The contractions were hard and consistent throughout the day, but by no means unbearable. Following the advice of my midwife, I remained mentally present in my labor, keeping my "nursing brain" out of it, never stopping to wonder how far dilated I might be or when it would be time to push. I simply breathed, rocked, and swayed my way through each contraction, focusing solely on the rhythm of my movements and the power of my laboring body.

By late afternoon, after nearly 15 hours of regular contractions, I sensed that my labor had changed. We had spent the early afternoon walking around an indoor mall, during which my contractions increased in frequency from about 8 minutes apart to 3 to 6 minutes apart. We came home, and I immediately made my way to the bathroom, overwhelmed by two or three very long, strong contractions. Looking for relief, I crawled into a hot shower while instructing my husband to call the midwife. I could feel my labor intensify with transition. After a brief phone call with the midwife, my husband and I decided to head over to the birth center. With help from my husband, I used short, quick breaths to help me stay relaxed through the most intense contractions. Twenty-five minutes later, Katie, our midwife, greeted us at the door of the birth center as I toddled my way into the center. Between contractions, I felt calm and focused, even conversational.

On the four-post bed of the labor room, which looked nothing like the hospital labor rooms I was accustomed to, Katie confirmed by digital exam what I already knew: I was completely dilated with just an anterior lip. I continued to labor at the center. An hour and a half later, it was time to push. With my first push, my water broke, bringing the baby's head far down into my pelvis. Even then, with the baby at plus 1 station, I didn't feel a tremendous urge to push. We pushed anyway, and in many different
I immediately wiped her dry and placed her on my chest to greet this

little life in my arms. In that moment, our lives changed forever.

positions: on the toilet, on my side, on hands and knees, and in a squatting position. Finally, in hope of getting the baby's head to slip under my pubic bone, I asked to push in a semisitting position, which did the trick. At 9:01 p.m., June Dahlia Bauer was born. The midwife placed her on my chest, and I immediately wiped her dry and placed her on my chest to greet this little life in my arms. In that moment, our lives changed forever.

In just 8 short weeks after June's birth, I returned to work full time: three night shifts a week. Initially, I was afraid that I would judge those who chose to use epidural anesthesia, given that I had labored on my own without medication easily enough. I was also apprehensive about returning to a birth culture that embraced active management of the labor process. Instead, my personal experience with labor and birth transformed me on the unit floor. As I gain strength in my clinical skills, I continue to draw from my own experience to better advocate for my laboring patients. For example, I steer my patients' focus away from the pain of contractions by encouraging them to use imagery and rhythm. I also encourage movement in labor, even for those who are confined to a bed following epidural anesthesia. When it comes time to push, I dim the lights in the room and ask the residents and medical staff to use soft voices to maintain a calm and centered ambiance. Most importantly, I have learned that patient education is central to my role as an advocate for normal birth. I put more energy and time into reminding my patients to question medical interventions that seem unnecessary or routine. By doing this, I believe I am better serving my patients and helping women find their own way through labor and birth in the hospital setting.

AMY BAUER is a labor and delivery nurse in Washington, $D C$, where she lives with her husband, daughter, and darling pug, Pearl. Prior to her nursing career, Bauer worked for Lamaze International as the education program coordinator. When she is not helping women in labor or caring for her beloved daughter, June, she enjoys knitting and baking for anyone interested.

I have learned that patient education is central to my role as an advocate for normal birth. 